\title{
Emotion regulation predicts change of perceived health in patients with rheumatoid arthritis
}

\author{
H van Middendorp, R Geenen, M J Sorbi, L J P van Doornen, J W J Bijlsma
}

Ann Rheum Dis 2005;64:1071-1074. doi: 10.1136/ard.2004.020487

\begin{abstract}
Objectives: To examine whether emotion regulation predicts change of perceived health in patients with rheumatoid arthritis (RA).

Methods: Sixty six patients (44 female, 22 male; mean (SD) age 57.7 (11.6) years) participated in a prospective study. Hierarchical regression analysis was used to predict change of perceived health between study entry and follow up $\left(1 \frac{1}{2}\right.$ years later) from the emotion regulation styles ambiguity, control, orientation, and expression at study entry.

Results: Valuing and intensely experiencing emotions (emotional orientation) predicted a decrease of positive affect. Difficulty recognising and expressing emotions (ambiguity) predicted an increase of perceived disease activity. Emotion regulation showed no associations with change of negative affect and social and physical functioning.

Conclusions: Two styles of emotion regulation were shown to have a significant though modest role in the prediction of perceived health change in patients with RA. This suggests that the monitoring of emotion regulation may help to identify patients who are at risk for a reduction of perceived health. If our findings were confirmed by experimental research, improving emotion regulation skills might favourably affect perceived health.
\end{abstract}

$\mathrm{R}$ heumatoid arthritis (RA) has consequences for perceived psychosocial and somatic health. Diathesis-stress models propose that biomedical and disease related stressors, in combination with specific internal vulnerability factors of the person, affect perceived health. ${ }^{1}$ Person characteristics like cognitions and behavioural coping styles have been shown to predict perceived health in RA. ${ }^{2}$ The role of emotions in health and disease has been recognised for centuries as a fundamental ingredient of psychological adaptation, but has been rather neglected in psychological research. Lately, emotion regulation has been receiving increasing attention as a relevant addition to existing psychological concepts on the interface between psychology and health..$^{3-10}$ Emotion regulation refers to the processes by which individual people influence which emotions they have, when they have them, and how they experience and express these emotions. ${ }^{3}$ Examples of emotion regulation strategies are whether people can recognise and describe their emotions, the intensity of experiencing emotions, and whether they tend to express or suppress their emotions. Individual patients differ in their use of emotion regulation strategies in adjusting to the burden of their disease, and these individual differences may have implications for perceived health. ${ }^{4}$

Prospective studies in healthy and chronically ill populations, including cancer, coronary heart disease, and HIV, but not RA, have shown that aspects of emotion regulation can predict change of health status. Emotional expression was related to improved health and psychological wellbeing, ${ }^{5}$ whereas emotionally inexpressive coping was related to disease progression. ${ }^{6}$ Attending to and valuing emotions in daily life and decision making was related to an increase in distress in patients with breast cancer. ${ }^{7}$ Difficulty in identifying and describing emotions, alexithymia, was predictive of a decrease in psychological and physical functioning, all-cause mortality, and a poor treatment outcome. ${ }^{8}$ Experimental studies showed that health outcome improved after alexithymia decreased ${ }^{9}$ and emotional expression was encouraged. ${ }^{10}$

Perceived health is a relevant aspect of functioning in RA that may influence, among other things, help seeking behaviour, symptom report, and adherence to drug treatment. Knowledge of the ability of emotion regulation to predict change of perceived health offers insight into possible protective and risk factors for a reduction of perceived health, and may offer suggestions to affect perceived health supplementary to regular health care. A previous cross sectional study by our group showed that the way in which patients with RA regulate their emotions was associated with the way they perceived their health. ${ }^{11}$ This prospective study aimed at examining whether emotion regulation predicts change of perceived health in patients with RA.

\section{METHODS}

Styles of emotion regulation and perceived health were assessed at study entry in 338 outpatients with RA for a cross sectional questionnaire study. At a mean (SD) follow up assessment 15 (4) months later, perceived health was assessed again before the start of an intervention study on emotional disclosure in 66 of these patients. Both studies were approved by the research and ethics committee of the University Medical Centre, Utrecht. Table 1 reports patient characteristics at study entry. Compared with the remainder of the 338 patients, the 66 patients who volunteered for this study were characterised by less frequent use of analgesics, more frequent use of non-steroidal anti-inflammatory drugs, and higher scores on emotional orientation and positive affect. No differences were seen for the other variables.

Four questionnaires were administered to assess how individual patients generally respond to emotional situations. Subsequently, principal component analysis on the 14 scales of these questionnaires was used to extract four aspects of emotion regulation ${ }^{11}$ :

- Ambiguity: a combination of alexithymia and ambivalence on expressing emotions

- Control: keeping feelings inside and trying to restrain feelings and be rational despite experiencing emotions

Abbreviations: DAS28, 28 joint count Disease Activity Score; RA, rheumatoid arthritis 


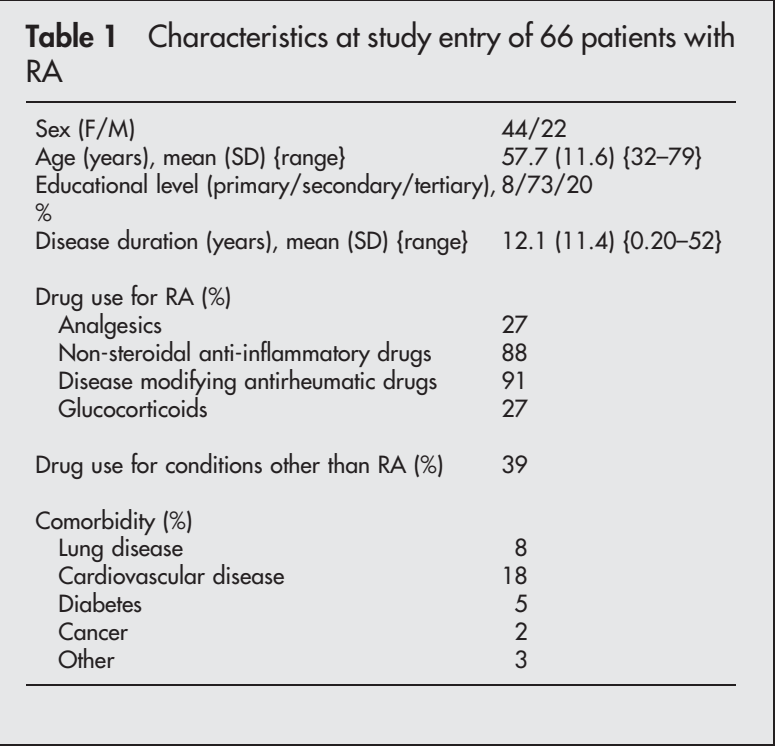

- Orientation: attending to and valuing emotions in daily life and decision making, and experiencing emotions intensely

- Expression: the expression of both negative and positive emotions towards others.

The factor solution was clearly interpretable by high loadings of most scales on a single factor and the internal consistency of the styles of emotion regulation varied between $\alpha=0.63$ for orientation and $\alpha=0.79$ for ambiguity. ${ }^{11}$ At study entry and follow up, five aspects of perceived health were assessed, extracted from principal component analysis on 16 scales of four self report instruments ${ }^{11}$ :

- Negative affect: depressed and tense mood

- Positive affect: energetic and cheerful mood

- Social functioning: actual and perceived social support

- Physical functioning: self care, disability, and mobility

- Disease activity: pain and self assessed disease activity.

The Rheumatoid Arthritis Disease Activity Index (RADAI) ${ }^{12}$ was used to measure self assessed disease activity. The internal consistency of these aspects varied from $\alpha=0.59$ for social functioning to $\alpha=0.91$ for negative affect and disease activity.

A clinical measure of disease activity at follow up was composed of the erythrocyte sedimentation rate and 26 tender and swollen joint counts (excluding the shoulders), which gives at most a small underestimation of the 28 joint count Disease Activity Score (DAS28). ${ }^{13}$

To examine whether perceived health was related to clinical disease activity, Pearson correlation coefficients were calculated. The covariates which had to be controlled were determined and the emotion regulation styles which had predictive potential were established. To this aim, both the potential covariates (number of days between study entry and follow up, age, sex, educational level, disease duration, and comorbidity) and the styles of emotion regulation were correlated with the residual change scores of perceived health. These residual change scores were the scores for perceived health at the follow up adjusted for initial values of perceived health. Hierarchical regression analyses were performed on the health aspects at follow up to examine the relative contribution of the styles of emotion regulation to change in perceived health. After taking into account the initial values on the dependent variables (step 1) and

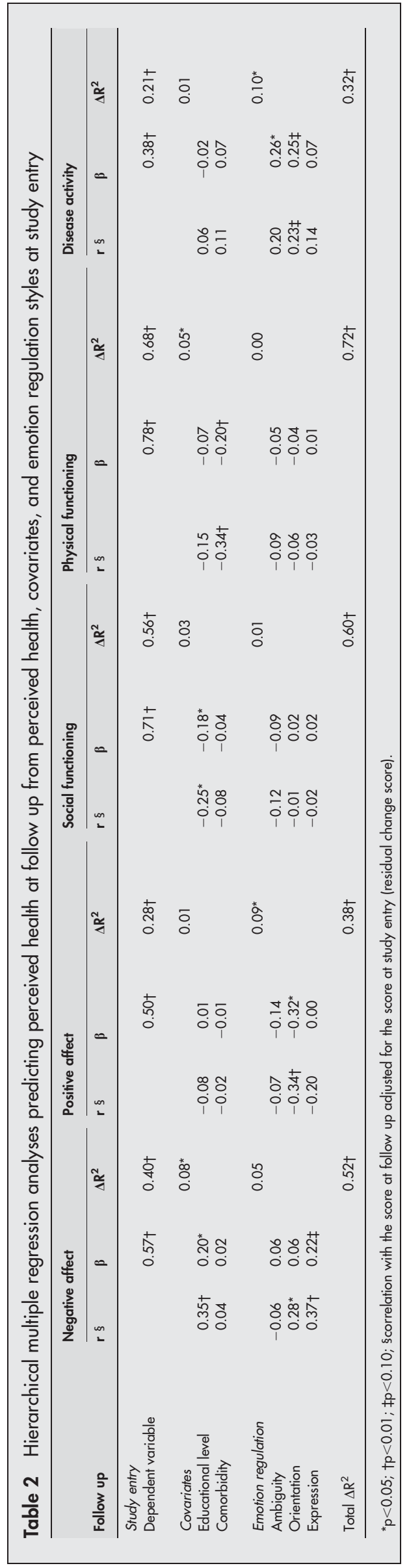



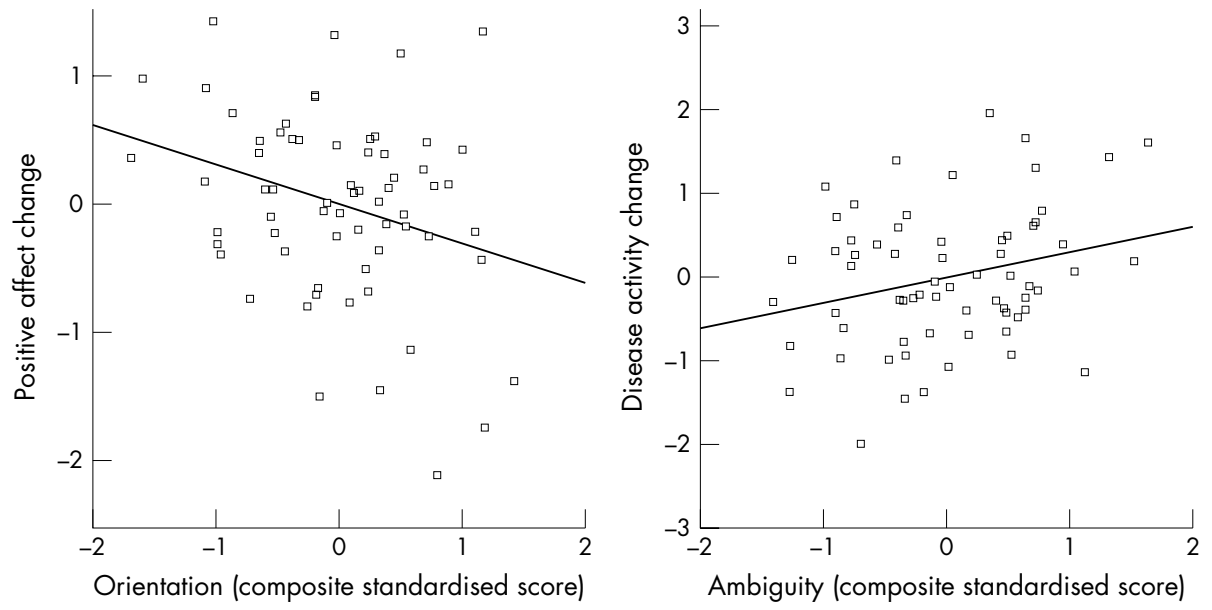

Figure 1 Scatter plots of the relation between orientation and the residual change score of positive affect, and between ambiguity and the residual change score of disease activity.

covariates (step 2), the styles of emotion regulation were entered ( step 3).

\section{RESULTS}

At follow up, the clinical measure of disease activity (DAS28) showed moderate to high correlations with the self report measures of disease activity $(r=0.39, \mathrm{p}=0.001)$ and physical functioning $(r=-0.57, \mathrm{p}=0.000)$, and no significant correlation with the two affect measures and social functioning.

Controlling one's emotions was unrelated to the residual change scores of health and was omitted from subsequent analyses. Initial values for perceived health were the best predictor of perceived health at follow up (table 2). Social and physical functioning, especially, showed stability.

Emotion regulation predicted change of positive affect $(F$ change $(3,59)=2.95, \mathrm{p}=0.040)$ and perceived disease activity $(F$ change $(3,59)=2.84, \mathrm{p}=0.045)$. The higher patients scored on orientation, the more their positive affect had decreased at follow up $(t(59)=-2.35, \mathrm{p}=0.022)$ (fig 1$)$. Low scores for ambiguity tended to be associated with a decrease of perceived disease activity, and high scores with an increase $(t(59)=2.22, \mathrm{p}=0.030)$ (fig 1). Also, orientation was a marginally significant predictor of a change of perceived disease activity $(t(59)=1.76, \quad \mathrm{p}=0.083)$. Expression was significantly correlated with change of negative affect, but the association became marginally significant after controlling for covariates and the other styles of emotion regulation. Change of negative affect and social and physical functioning was not significantly predicted by emotion regulation (table 2 ).

\section{DISCUSSION}

In our prospective study a decrease of positive affect and an increase of perceived disease activity in patients with RA were predicted by the emotion regulation styles orientation and ambiguity. Change of negative affect and social and physical functioning were not predicted by emotion regulation.

In line with a prospective study in patients with breast cancer, ${ }^{7}$ emotional orientation, attending to and valuing emotions, and experiencing them intensely, was shown to be a potential risk factor for psychological distress and perceived disease activity. This result differs from studies in populations that do not have a chronic disease, which report besides negative also positive associations of emotional orientation with health. ${ }^{14} 15$ Our findings support a diathesis-stress model: being emotionally sensitive appears to be a vulnerability factor for a disadvantageous health change in combination with the stress of a chronic and disabling disease.

Ambiguity was a significant but modest predictor of increased perceived disease activity, corresponding with previous studies. ${ }^{89}$ Ambiguity, which is conceptually closely linked to alexithymia, has been associated with the report of somatic distress and may delay treatment seeking. ${ }^{6}{ }^{9}$ Because symptoms may be inaccurately recognised as part of the disease or ineffectively communicated to rheumatologists, high ambiguity may hamper disease control.

That both emotional ambiguity and orientation showed some association with perceived disease activity corresponds with hypotheses on the physiological concomitants of emotion regulation. ${ }^{16}$ Experiencing emotions intensely and valuing emotions (orientation) as well as inhibiting or suppressing emotion response tendencies (ambiguity) may lead to exaggerated physiological arousal in an already compromised physiological system as in RA. Future research including neuroendocrine and immune variables may elucidate potential physiological mediational pathways linking emotion regulation and health.

Disease activity measures showed moderate to high correlations with perceived health. This suggests that change in perceived health in our study reflects actual disease activity besides psychological influences. Because disease duration in our sample was unrelated to our findings, the suggested impact of emotion regulation on perceived health outcome does not seem restricted to the first adjustment phase of the disease.

A limitation of our study is that the causal pathway from emotion regulation to change of perceived health cannot be ascertained. Emotion regulation was assessed only at study entry, preventing reciprocal effects from being studied. The possibility cannot be excluded, for example, that a person with decreased perceived health has been deteriorating over a longer period of time, which may have led to emotional ambiguity or orientation. It may also be that the predictive associations are the consequence of some unmeasured third variable. Other methodological restrictions are the relatively small sample size, and possibly reduced generalisability because only volunteers of an emotionally oriented intervention participated. Confirmation studies in a larger sample, measuring both emotion regulation and health repeatedly over time, are needed. In such a design the strength of different directions of relationships can be examined by structural equation modelling. 
In conclusion, two styles of emotion regulation were shown to have a significant though modest role in the prediction of perceived health change in patients with RA. This suggests that the monitoring of emotion regulation may help to identify patients who are at risk for a reduction of perceived health. If our findings were confirmed by experimental research, improving emotion regulation skills might favourably affect perceived health.

\section{ACKNOWLEDGEMENTS}

This study was financially supported by the Dutch Arthritis Association. We thank all rheumatologists and rheumatology nurses of the Arthritis Research Foundation Utrecht (SRU) for recruitment of participants, and Etiënne Blaas, Hanna Zijlstra, Tanja van Meerveld, Nettie den Breejen, Femke Vergeer, Ariëtte van Wijngaarden, Jolien Spoelstra, Nicole Dreessen, Ruth Haakman, and Annechien van den Bosch for help in data collection.

\section{Authors' affiliations \\ $H$ van Middendorp, R Geenen, M J Sorbi, L J P van Doornen}

Department of Health Psychology, Utrecht University, The Netherlands J W J Bijlsma, Department of Rheumatology and Clinical Immunology, University Medical Centre, Utrecht, The Netherlands

Correspondence to: $\mathrm{Dr} \mathrm{H}$ van Middendorp, Department of Health Psychology, Utrecht University, Heidelberglaan 1, PO Box 80.140, 3508 TC Utrecht, The Netherlands; H.vanMiddendorp@fss.uu.nl

Accepted 18 December 2004

\section{REFERENCES}

1 Huyser B, Parker JC. Stress and rheumatoid arthritis: an integrative review. Arthritis Care Res 1998;11:135-45.

2 Evers AW, Kraaimaat FW, Geenen R, Jacobs JW, Biilsma JW. Stressvulnerability factors as long-term predictors of disease activity in early rheumatoid arthritis. J Psychosom Res 2003;55:293-302.
3 Gross JJ. The emerging field of emotion regulation: an integrative review. Rev Gen Psychol 1998;2:271-99.

$4 \mathrm{Gohm}$ CL, Clore GL. Four latent traits of emotional experience and their involvement in well-being, coping, and attributional style. Cogn Emotion 2002:16:495-518.

5 Eng PM, Fitzmaurice G, Kubzansky LD, Rimm EB, Kawachi I. Anger expression and risk of stroke and coronary heart disease among male health professionals. Psychosom Med 2003:65:100-10.

6 Solano L, Costa M, Temoshok L, Salvati S, Coda R, Aiuti F, et al. An emotionally inexpressive (type C) coping style influences HIV disease progression at six and twelve month follow-ups. Psychol Health 2002; 17:641-55

7 Stanton AL, Danoff-Burg S, Cameron CL, Bishop M, Collins CA, Kirk SB, et al. Emotionally expressive coping predicts psychological and physical adjustment to breast cancer. J Consult Clin Psychol 2000;68:875-82.

8 Porcelli P, Bagby RM, Taylor GJ, De Carne M, Leandro G, Todarello O. Alexithymia as predictor of treatment outcome in patients with functional gastrointestinal disorders. Psychosom Med 2003;65:911-18.

9 Beresnevaite M. Exploring the benefits of group psychotherapy in reducing alexithymia in coronary heart disease patients: a preliminary study. Psychother Psychosom 2000;69:117-22.

10 Lepore SJ, Smyth JM, eds. The writing cure: how expressive writing promotes health and emotional well-being. Washington, DC: American Psychological Association, 2002.

11 Van Middendorp H, Geenen R, Sorbi MJ, Hox JJ, Vingerhoets AJM, Van Doornen LP, et al. Styles of emotion regulation and their associations with perceived health in patients with rheumatoid arthritis. Ann Behav Med, (in press).

12 Stucki G, Liang MH, Stucki S, Bruhlmann P, Michel BA. A self-administered rheumatoid arthritis disease activity index (RADAl) for epidemiologic research. Psychometric properties and correlation with parameters of disease activity. Arthritis Rheum 1995;38:795-8.

13 Prevoo MLL, Van 't Hof MA, Kuper HH, Van Leeuwen MA, Van de Putte LBA, Van Riel PLCM. Modified disease activity scores that include twenty-eight-joint counts. Arthritis Rheum 1995;38:44-8.

14 Gross JJ, John OP. Mapping the domain of expressivity: multimethod evidence for a hierarchical model. J Pers Soc Psychol 1998;74:170-91.

15 Stanton AL, Kirk SB, Cameron CL, Danoff-Burg S. Coping through emotional approach: Scale construction and validation. J Pers Soc Psychol 2000;78:1150-69.

16 Lepore SJ, Greenberg MA, Bruno M, Smyth JM. Expressive writing and health: self-regulation of emotion-related experience, physiology, and behavior. In: Lepore SJ, Smyth JM, eds. The writing cure: how expressive writing promotes health and emotional well-being. Washington, DC: American Psychological Association, 2002:99-117. 